\title{
Testing a Heuristic Algorithm for Finding a Maximum Clique on DIMACS and Facebook Graphs
}

\author{
VLADIMIR BALASH \\ Saratov State University \\ Faculty of Mechanics and Mathematics \\ 83 Astrakhanskaya Street \\ RUSSIAN FEDERATION \\ vladimirbalash@yandex.ru \\ DANIIL VOLKOV \\ Saratov State University \\ Faculty of Computer Science and IT \\ 83 Astrakhanskaya Street \\ RUSSIAN FEDERATION \\ volkovda351@gmail.com
ALEXEY FAIZLIEV
Saratov State University
Risk Institute
83 Astrakhanskaya Street
faizlievar1983@mail.ru \\ RUSSIAN FEDERATION
}

\author{
ANASTASIA STEPANOVA \\ Saratov State University \\ Risk Institute \\ 83 Astrakhanskaya Street \\ RUSSIAN FEDERATION \\ stacey.stepanova@gmail.com
}

\author{
SERGEI MIRONOV \\ Saratov State University \\ Faculty of Computer Science and IT \\ 83 Astrakhanskaya Street \\ RUSSIAN FEDERATION \\ mironovsv@info.sgu.ru
}

\author{
SERGEI SIDOROV \\ Saratov State University \\ Risk Institute \\ 83 Astrakhanskaya Street \\ RUSSIAN FEDERATION \\ sidorovsp@yahoo.com
}

\begin{abstract}
In this paper we propose a new heuristic algorithm for solving a maximum clique search problem (MCP). While the proposed algorithm (called TrustCLQ) uses a general approach to solving MCP, it is almost independent of the order of vertices and does not exploit a partition of the graph into independent sets. The algorithm was tested on DIMACS library graphs which are often employed for testing MCP solution algorithms. TrustCLQ algorithm was compared with the well-known ILS heuristic algorithm (as well as with a standard algorithm from networkx library)onDIMACSdatasets.Moreover,TrustCLQalgorithmhasbeentestedonFacebooksocialgraphs.
\end{abstract}

Key-Words: Heuristic algorithm, Graphs, Cliques, Network analysis, Maximum clique problem, DIMACS graphs, Socialgraphs

Received: November 3, 2020. Revised: March 26, 2020. Accepted: April 10, 2020. Published: April 30, 2020.

\section{Introduction}

The Maximum Clique Problem (MCP) is one of the most well-known NP-hard problems in graph theory [1]. A clique in a graph is a subset of vertices that form a complete subgraph. A maximum clique in a graph is the maximum-sized subset of such vertices.

Applications of the maximum clique problem are quite wide. In bioinformatics, MCP is used in computer analysis of genomic databases, e.g. in the search for potential regulatory structures of ribonucleic acids. In social networks, MCP is used for clustering data, e.g. for dividing various communities into groups (clusters) that share common properties. Cluster al-

E-ISSN: 2224-2856 location allows each of them to be processed by a separate auxiliary server. In chemistry, the MCP problem underlies the search for the maximum general substructure in the graph describing the structure of the chemical compound. In addition, MCP is a mathematical model of a number of problems arising in the electronic equipment design.

In these applications, exact MCP solutions are usually required. However, the size of input data is usually too huge (input graphs can contain up to a million vertices). Thus, the important topic of MCP research is the development of new approaches for finding MCP solutions taking into account the features of 
graphs that arise in applications.

Let $G=(V, E)$ be an undirected graph, where $V=\{1, \ldots, n\}$ is the set of vertices and $E \subset V \times V$ is the set of edges. Let $G(S)=(S, E \cap S \times S)$ denote the subgraph formed by the subset of vertices $S$. The notion $\bar{G}$ will be used for the complement of the graph $G, \bar{G}=(V, \bar{E})$, where $\bar{E}=\{(i, j) \mid i, j \in V, i \neq$ $j$ and $(i, j) \notin E\}$. Two vertices $i$ and $j$ are adjacent if $(i, j) \in E$. The neighbors of vertex $i$ are the set of vertices adjacent to $i, N(i)=\{j \in V:(i, j) \in E\}$. Let $A_{G}$ denote the adjacency matrix of graph $G$; the adjacency matrix has size $n \times n, A_{G}=\left(a_{i j}\right)$, where $a_{i j}=1$ if $(i, j) \in E$ and $a_{i j}=0$, otherwise. The degree $\operatorname{deg}(i)$ of vertex $i$ in $G$ is defined as the number of neighbors of vertex $i$ in $G$ and will be denoted by $|N(i)|$. Let $\delta(G)$ and $\Delta(G)$ denote the least and greatest degree of vertices in $G$, respectively.

A graph $G=(V, E)$ is called complete if all its vertices are adjacent to each other, i.e. if $\forall i, j \in V$, $i \neq j,(i, j) \in E$. We define a clique $C$ as a subset of vertices such that $G(C)$ is complete. An independent set in $G$ is a subset of the vertices of $I$ such that the subgraph $G(I)$ formed by them does not contain edges. A clique (independent set) is called maximum if there is no larger clique (independent set) in the graph.

The clique number is the number of vertices in the maximum clique in $G$ and traditionally denoted by $\omega(G)$. The independence number $\alpha(G)$ is defined similarly. It is easy to see that $C$ is a maximum clique in $G$ if and only if $C$ is a maximal independent set in $\bar{G}$, therefore $\omega(G)=\alpha(\bar{G})$.

There are several dozens of algorithms for solving MCP which can be classified either exact algorithms or heuristic ones. Exact algorithms are usually based on exhaustive search in conjunction with the branch-and-bound method or different vertexcoloring schemes [2]. The exact algorithms such as the MCQ algorithm [3], MCR [4], MCS [5], MaxCliqueDyn [6], MaxCliquePara (MCP) [7], BBmaxClique [8], FastMaxClique (FMC) [9], parallel maximum clique (PMC) algorithm [10], an implementation of a MotzkinStraus-based iterative clique-finding algorithm for GPUs [11], among many others, have proved their applicability in solving MCP. One of the currently best exact approaches was proposed in the paper [12].

Heuristic algorithms employ a wide variety of ideas including reactive local search [13], randomness in DLS [14] and CLS algorithms [15], a deterministic greedy heuristic that adds vertices in an order depending on their weights in UALEX-MS algorithm [16], a hybrid evolutionary method in EAG [17]. Often, specific properties of the studied graph are used, e.g. a special order of vertices, the known lower and upper bounds, the chromatic number of a graph, etc. Exact algorithms are ineffective in solving NP-complete problems, since they are based on exhaustive search. While heuristic algorithms are not accurate, they may be more promising in real applications, since they obtain the solution in much lesser time. Heuristic algorithms all differ in accuracy, speed, and they often use specific properties of graphs. One of the currently best heuristic methods is LSCC+BMS, proposed in the paper [18].

The recent development on $\mathrm{MCP}$ can be found in papers [19, 20, 21, 22, 23, 24, 25, 26, 27, 28].

In this paper, one of such algorithms is proposed and examined. We present a novel simple iterative algorithm for maximum clique finding. The standard iterative approach is extended by using the trust indicator of vertices. The proposed method belongs to the class of heuristic optimization methods. Therefore, the proposed algorithm may be convenient for solving many real-world problems related to large social networks.

In this paper, the proposed algorithm is compared with the ILS heuristic algorithm [29] as well as with a standard algorithm implemented in the networkx library https://networkx.github.io (max_clique(), below nx.max_clique functions) on DIMACS data sets. The empirical results show that the TrustCLQ algorithm performs better than the standard algorithm from networkx library. Moreover, TrustCLQ algorithm has been tested on Facebook social graphs.

\section{Trust-Based Algorithm (Trust- CLQ)}

The main ideas used in heuristic algorithms include vertex cut-off, independent set cut-off, centralization coefficient selection, pseudo-random selection. The proposed algorithm is based on some of these ideas. An effective fast heuristic should combine as many strengths as possible (including accuracy) and as few weaknesses as possible (e.g. execution time). Moreover, the rejection of one or another approach should provide clear advantages. It is not possible to obtain high accuracy without significant loss of time, because one way or another it all comes down to a complete enumeration of all branches of the decision tree. 


\subsection{Vertex trust indicator}

The new approach in the MCP solution proposed in this paper will be based on the use of the trust indicator for each vertex in the graph. It has the following intuitive practical meaning. If we expected that the vertex $v$ will form a clique of size $k$, but it has not happened at an iteration, then we decrease confidence in the vertex. Since vertices with a greater degree are less likely to form a clique of size $k$ on the first attempt, the trust in them should melt away more slowly. In this paper we propose to evaluate the vertex trust indicator by

$$
\operatorname{trust}(v)=\operatorname{trust}(v)-\frac{k}{\operatorname{deg}(v)+1}
$$

at each iteration of the algorithm.

Note that the vertex trust indicator may also incorporate a dependence on the binomial coefficient, since the maximum number of cliques of size $k$ in a graph with $n$ vertices is equal to $\frac{n !}{k !(n-k) !}$. However, due to the sparseness of real graphs, the number of possible cliques is much less than expected. For this reason, we use a very simplified and approximate method of evaluating a vertex trust defined by equation (1). As will be shown below, this approach showed acceptable accuracy on sparse graphs.

The initial value of the trust indicator of each vertex can be set to 1 . This indicator can be changed before running the algorithm. The higher the value of the trust indicator for a vertex, the higher should be the accuracy of the solution obtained by the algorithm, since a larger value of the trust indicator increases the number of iterations, each of which has a chance to improve the solution.

This method allows anyone to search for maximum cliques in graphs about which nothing is known in advance. The algorithm does not use the concepts of graph coloring and the number of its independent sets. In this sense, the proposed approach is more universal. At the same time, one can adjust the accuracy of the algorithm by changing the initial value of vertex trust indicator. If one needs to get an approximate solution on a large graph, then the initial value of vertex trust indicator can be set to minimum. If one need to obtain the most possible accurate solution, then the indicator should be set higher.

Obviously, if there is a clique of size $k$ in the graph, then there is also a clique of size $k-1$. It would be logical that one should not continue to search for large cliques in the absence of smaller ones. However, this is only correct with respect to exact algorithms.
When it comes to our heuristic algorithm, then equation (1) comes into play to find an heuristic evaluation of each vertex. As one can see, with an increase in the size of the clique $k$, the number of possible cliques decreases. Therefore, by cutting off vertices with a small degree, such algorithms are assumed to be able to demonstrate a good accuracy. Thus, the probability of finding the best solution may be increasing.

\subsection{Vertex removal}

One of the elements of our algorithm is the removal of irrelevant vertices. It may work in a positive way, since it reduces the size of the search tree and increases the likelihood of finding the optimal solution. In our algorithm, we will remove a vertex in two cases.

- In the first case, the vertices are removed using the degree-based criterion. Suppose we are looking for a clique of size $k$ in a graph. Then all vertices with degree $\operatorname{deg}(v)+1<k$ should be permanently removed from the graph. This will not affect the solution, but only allow it to be improved due to the fact that the "useless" vertices will not affect the size of the search tree.

- In the second case, the vertices are removed when a confidence in the vertex is lost. If the trust indicator (1) of vertex $v$ at a certain iteration becomes less than zero, i.e. $\operatorname{trust}(v)<0$, then the vertex is removed from the graph.

\subsection{TrustCLQ algorithm}

The proposed algorithm (called TrustCLQ algorithm) consists of several functions. The main function $\mathrm{f}$ indMaxClique accepts an undirected and unweighted graph without multi-edges and loops. Vertex numeration starts from zero. If nothing is known about the upper border of the clique, then we can safely assume that it is equal to the number of vertices in the graph. If the lower boundary is unknown, then it is equal to 1 or $\delta(G)$, i.e. the least degree of vertices in $G$. The initial solution is the empty set. The pseudocode of $\mathrm{findMaxClique}$ function can be found in Algorithm 1. The function moves from the bottom boundary to the top boundary. If a clique of size $i$ is searched, then the function immediately removes from the graph all vertices with degree $\operatorname{deg}(v)+1<i$. They will no longer be considered in the algorithm execution. Vertices from the graph are removed using the reduceVertex function (Algorithm 4). 
Then the main function $\mathrm{findMaxClique} \mathrm{runs}$ the clique search function named findClique on the updated graph and update our solution. The pseudocode of function $\mathrm{findClique}$ is presented in Algorithm 2. The function directly chooses a solution and calculates the trust indicator (1) for each vertex. The global variable SCALE is responsible for that and its value can be changed before each run of the algorithm.

Function $f i n d C l i q u e$ takes the next vertex and tries to build a solution that will be no worse than the already known solution, i.e. it should be a clique of size at least $L B$. For each vertex, a list of candidates is formed that can be included in the solution. The Bron-Kerbosch algorithm is employed to perform a complete search of all possible variations of candidates. At each iteration, TrustCLQ selects one of them randomly. This candidate is added to the solution without any checks, since the meaning of the list of candidates is that they are all suitable for inclusion to the solution. After that, the list is updated to the current one.

The update function updatecandidates is presented in Algorithm 3. While this reduces the search tree to a minimum, it reduces the accuracy. If the solution has been improved, then control is transferred back to function $\mathrm{f}$ indMaxClique.

An important feature of the algorithm is that it allows to control the accuracy by changing the initial value of the trust indicator (the SCALE parameter). For example, with the initial value of the trust indicator of $\frac{1}{n}$, where $n$ is the number of vertices in the graph, the algorithm has low accuracy. However, with an increase of its value, the accuracy increases (of course, along with the running time of the algorithm). One can choose between a high initial value of the trust indicator, which leads to a higher running time, but provides a solution close to optimal, or a low initial value of the trust indicator, which gives a quick but an inaccurate result.

Note that the algorithm does not require a significant amount of additional memory to work. It stores only a local copy of the graph and several sets whose cardinality does not exceed the number of the graph vertices.

The running time is difficult to estimate, since it also depends on the internal structure of the graph. In the course of the empirical study, it could be noted that the average value of the vertex degree has a great influence on the running time. Indeed, despite the fact that there are a lot of links in social graphs, they are processed by the algorithm quite quickly, since their density is very low.

The algorithm can also be used to solve the problem of an independent set finding. In this case, it is necessary to take the complement of the graph and submit it as the input to the algorithm.

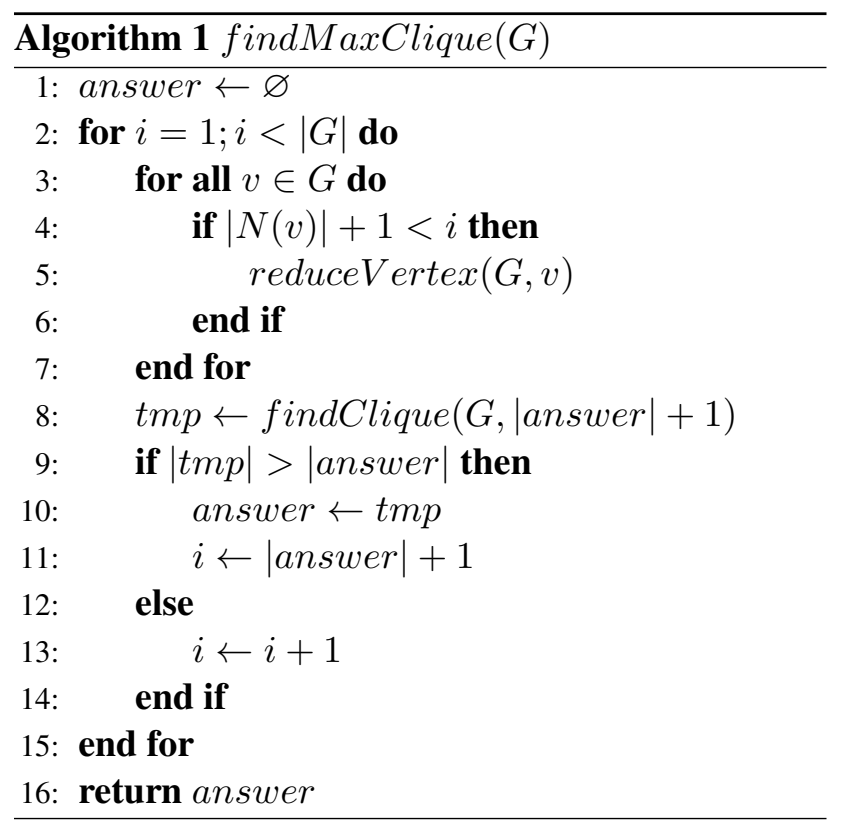

If the solution has not been improved at an iteration, then the trust indicator of the vertex, from which this solution was formed, decreases according to equation (1). If the value of the trust indicator of the vertex becomes negative, then the vertex is removed from the graph. If the algorithm does not find a clique that improves the solution, then it returns the empty set. However, this does not unambiguously indicate that a maximum clique has been found. The solution can still be improved by cutting off another vertices and restarting the algorithm.

The algorithm was implemented in Python and its code can be found by link https://github. $\mathrm{com} / \mathrm{stacy}-\mathrm{s} / \mathrm{MCP}$. All runs were carried out on the Amazon EC, Intel Xeon Platinum 8000 series (Skylake-SP) $3.1 \mathrm{GHz}, 2 \mathrm{vCPU}, 1 \mathrm{~Gb}$.

\section{Empirical results}

\subsection{DIMACS graphs}

To test TrustCLQ algorithm, we will use several graphs from DIMACS benchmark set http://iridia.ulb.ac.be/ fmascia/ maximum_clique/DIMACS-benchmark, which are usually employed to test the algorithms designed to solve the maximum clique problem. All 


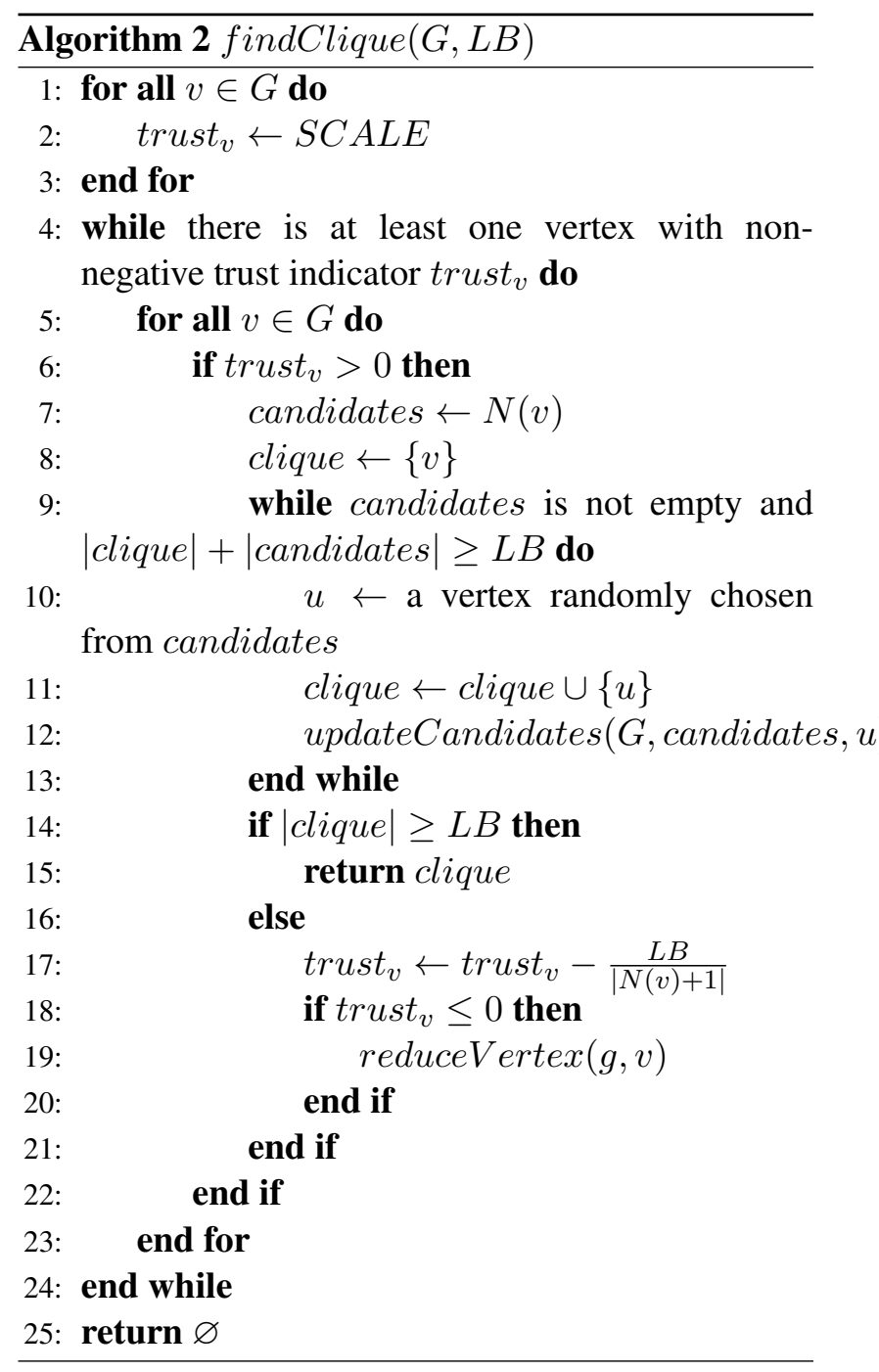

graphs differ from each other in their characteristics. The DIMACS benchmark set consists of the graphs that are widely used to test the solution for MCP [30]. All test graphs are constructed based on various principles. For example, graphs of the gen family are generated as follows: they take as a basis a complete subgraph of a known size and add the necessary number of edges to it.

Table 1 shows the results and running time for the TrustCLQ, nx.max_clique algorithms as well as the results for the ILS algorithm (results for the ILS algorithm were obtained in [31]).

The algorithm showed the most inaccurate results when using the initial trust indicator value of 0.01 . It can be explained by the fact that almost always after an unsuccessful construction of a clique, the vertex is removed from the graph, since the value of the trust indicator immediately becomes negative.
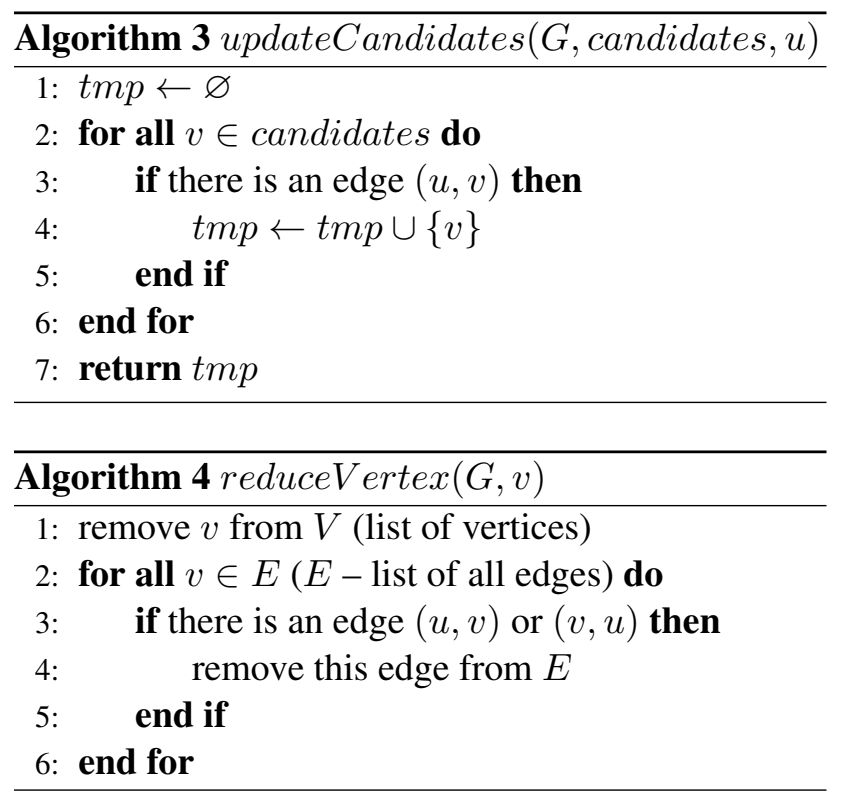

Empirical results presented in Table 1 shows that the TrustCLQ algorithm running time and its accuracy is increasing with an increase in the value of the SCALE parameter. The results also show that the TrustCLQ algorithm has a greater accuracy and a shorter running time than the nx.max_clique algorithm on almost all graphs from the DIMACS data set. Moreover, in some cases, the TrustCLQ algorithm obtains the exact solution and performs no worse than the ILS algorithm.

\subsection{The performance of the TrustCLQ algo- rithm on Social networks}

The portal [32] contains several hundred of different real networks arisen in a wide range of applied areas including biological networks, chemical structures, economic relationships, infrastructure and road networks, and social networks. The TrustCLQ algorithm was tested on dozens of Facebook graphs taken from [32].

These graphs are very different from DIMACS graphs in terms of the node degree distribution as well as the edge density. Table 2 presents some of the obtained results. It shows that even with a large number of nodes and edges, the algorithm works much faster than on DIMACS graphs. This is due to the much greater sparseness of social networks, since a persons ability to form social connections with other network participants is limited. In addition, in the social networks, the spread in the vertex degrees is greater than in random graphs. 


\begin{tabular}{|c|c|c|c|c|c|c|c|c|c|c|}
\hline & Dataset & $|V|$ & $t_{0.1}$ & $\omega_{0.1}$ & $t_{1.0}$ & $\omega_{1.0}$ & $\omega_{I L S}$ & $\omega_{\text {best }}$ & $t_{n x}$ & $\omega_{n x}$ \\
\hline 0 & C1000.9 & 1000 & 185.63 & 52 & 2015.27 & 54 & - & - & 149.5 & 47 \\
\hline 1 & C125.9 & 125 & 0.05 & 30 & 0.49 & 31 & - & - & 0.63 & 26 \\
\hline 2 & C2000.5 & 2000 & 322.74 & 14 & 2705.67 & 14 & - & - & - & - \\
\hline 3 & C250.9 & 250 & 0.38 & 38 & 11.37 & 39 & - & - & 3.52 & 33 \\
\hline 4 & C500.9 & 500 & 4.24 & 44 & 89.23 & 47 & - & - & 21.9 & 40 \\
\hline 5 & DSJC1000_5 & 1000 & 19.01 & 14 & 157.96 & 14 & - & - & 74.12 & 11 \\
\hline 6 & DSJC500_5 & 500 & 1.28 & 11 & 11.66 & 12 & - & - & 10.83 & 10 \\
\hline 7 & MANN_a27 & 378 & 8.27 & 123 & 61.86 & 123 & 126 & 126 & 10.66 & 104 \\
\hline 8 & MANN_a45 & 1035 & 507.19 & 335 & 6154.56 & 337 & 344 & 345 & 178.72 & 276 \\
\hline 9 & MANN_a9 & 45 & 0.0 & 15 & 0.01 & 16 & 16 & 16 & 0.05 & 13 \\
\hline 10 & brock200_1 & 200 & 0.08 & 18 & 1.54 & 19 & 21 & 21 & 1.21 & 16 \\
\hline 11 & brock200_2 & 200 & 0.04 & 9 & 0.56 & 12 & 12 & 12 & 0.96 & 8 \\
\hline 12 & brock200_3 & 200 & 0.05 & 14 & 0.72 & 13 & 15 & 15 & 1.07 & 11 \\
\hline 13 & brock200_4 & 200 & 0.07 & 13 & 0.82 & 14 & 17 & 17 & 1.15 & 12 \\
\hline 14 & brock400_1 & 400 & 2.07 & 21 & 26.44 & 22 & 23 & 27 & 7.74 & 18 \\
\hline 15 & brock400_2 & 400 & 2.78 & 20 & 19.19 & 21 & 24 & 29 & 8.05 & 18 \\
\hline 16 & brock400_3 & 400 & 1.88 & 20 & 31.33 & 22 & 24 & 31 & 7.56 & 19 \\
\hline 17 & brock400_4 & 400 & 2.96 & 21 & 19.03 & 22 & 26 & 33 & 7.59 & 18 \\
\hline 18 & brock800_1 & 800 & 16.81 & 17 & 181.63 & 18 & 19 & 23 & 44.56 & 15 \\
\hline 19 & brock800_2 & 800 & 17.41 & 17 & 175.39 & 18 & 20 & 24 & 45.0 & 16 \\
\hline 20 & brock800_3 & 800 & 20.56 & 18 & 162.28 & 18 & 19 & 25 & 44.15 & 16 \\
\hline 21 & brock800_4 & 800 & 15.47 & 17 & 173.13 & 18 & 19 & 26 & 44.15 & 16 \\
\hline 22 & c-fat200-1 & 200 & 0.01 & 12 & 0.01 & 12 & 12 & 12 & 2.56 & 12 \\
\hline 23 & c-fat $200-2$ & 200 & 0.01 & 24 & 0.02 & 24 & 24 & 24 & 2.11 & 24 \\
\hline 24 & c-fat $200-5$ & 200 & 0.03 & 58 & 0.05 & 58 & 58 & 58 & 1.98 & 58 \\
\hline 25 & c-fat500-1 & 500 & 0.06 & 14 & 0.07 & 14 & 14 & 14 & 43.89 & 14 \\
\hline 26 & c-fat500-10 & 500 & 0.29 & 126 & 0.55 & 126 & 126 & 126 & 22.49 & 126 \\
\hline 27 & c-fat500-2 & 500 & 0.08 & 26 & 0.09 & 26 & 26 & 26 & 39.65 & 26 \\
\hline 28 & c-fat $500-5$ & 500 & 0.15 & 64 & 0.22 & 64 & 64 & 64 & 29.28 & 64 \\
\hline 29 & gen200_p0.9_44 & 200 & 0.2 & 33 & 2.45 & 35 & 44 & 44 & 1.51 & 30 \\
\hline 30 & gen200_p0.9_55 & 200 & 0.19 & 34 & 2.43 & 42 & 55 & 55 & 1.76 & 37 \\
\hline 31 & gen400_p0.9_55 & 400 & 1.63 & 40 & 69.33 & 45 & 54 & 55 & 8.35 & 37 \\
\hline 32 & gen400_p0.9_65 & 400 & 1.7 & 41 & 60.5 & 45 & 64 & 65 & 9.11 & 38 \\
\hline 33 & gen400_p0.9_75 & 400 & 1.65 & 41 & 34.1 & 45 & 75 & 75 & 10.27 & 40 \\
\hline 34 & hamming $10-2$ & 1024 & 176.39 & 277 & 2391.11 & 307 & 473 & 512 & 887.33 & 512 \\
\hline 35 & hamming10-4 & 1024 & 104.68 & 31 & 1005.45 & 32 & - & - & 59.64 & 33 \\
\hline 36 & hamming6-2 & 64 & 0.0 & 32 & 0.02 & 32 & 32 & 32 & 0.18 & 32 \\
\hline 37 & hamming6-4 & 64 & 0.0 & 4 & 0.0 & 4 & 4 & 4 & 0.06 & 4 \\
\hline 38 & hamming8-2 & 256 & 0.68 & 96 & 10.61 & 111 & 128 & 128 & 11.17 & 128 \\
\hline 39 & hamming8-4 & 256 & 0.29 & 13 & 1.68 & 13 & 16 & 16 & 1.45 & 16 \\
\hline 40 & johnson 16-2-4 & 120 & 0.05 & 8 & 0.29 & 8 & 8 & 8 & 0.2 & 8 \\
\hline 41 & johnson32-2-4 & 496 & 11.38 & 16 & 122.29 & 16 & - & - & 5.15 & 16 \\
\hline 42 & johnson8-2-4 & 28 & 0.0 & 4 & 0.0 & 4 & 4 & 4 & 0.01 & 4 \\
\hline 43 & johnson8-4-4 & 70 & 0.0 & 11 & 0.02 & 14 & 14 & 14 & 0.09 & 14 \\
\hline 44 & keller4 & 171 & 0.05 & 11 & 0.46 & 11 & 11 & 11 & 0.65 & 9 \\
\hline 45 & keller5 & 776 & 29.24 & 23 & 255.78 & 25 & 27 & 27 & 33.44 & 20 \\
\hline 46 & keller6 & 3361 & 18566.02 & 47 & - & - & - & - & - & - \\
\hline 47 & p_hat1000-1 & 1000 & 3.39 & 9 & 3.32 & 9 & 10 & 10 & 64.41 & 8 \\
\hline 48 & p_hat $1000-2$ & 1000 & 17.1 & 34 & 17.32 & 34 & 44 & 46 & 60.79 & 28 \\
\hline 49 & p_hat $1000-3$ & 1000 & 46.68 & 46 & 47.93 & 46 & 67 & 68 & 97.47 & 46 \\
\hline
\end{tabular}

Table 1: Results and running time of TrustCLQ, nx.max_clique and ILS algorithms on the DIMACS graphs; $t_{n x}$ denotes the running time of the nx.max_clique algorithm, $t_{0.1}$ and $t_{1.0}$ denotes the running time of the TrustCLQ algorithm for $S C A L E=0.1$ and $S C A L E=1.0$, respectively; $\omega_{\text {best }}$ is the best-known solution; $\omega_{I L S}$ denotes the solution obtained by the ILS algorithm; $\omega_{0.1}$ and $\omega_{1.0}$ denote the solutions obtained by the TrustCLQ algorithm for $S C A L E=0.1$ and $S C A L E=1.0$, respectively 


\begin{tabular}{|l|l|l|l|l|}
\hline Dataset & $|V|$ & $t_{1.0}$ & $\omega_{1.0}$ & $\omega_{L B}$ \\
\hline socfb-American75.mtx & $6 \mathrm{k}$ & 40.57 & 39 & 9 \\
socfb-Amherst41.mtx & $2 \mathrm{k}$ & 5.98 & 21 & 10 \\
socfb-Auburn71.mtx & $18 \mathrm{k}$ & 663.72 & 56 & 13 \\
socfb-BC17.mtx & $12 \mathrm{k}$ & 200.13 & 35 & 12 \\
socfb-BU10.mtx & $20 \mathrm{k}$ & 483.46 & 38 & 12 \\
socfb-Baylor93.mtx & $13 \mathrm{k}$ & 319.47 & 54 & 11 \\
socfb-Berkeley13.mtx & $23 \mathrm{k}$ & 832.31 & 42 & 15 \\
socfb-Bingham82.mtx & $10 \mathrm{k}$ & 150.24 & 42 & 10 \\
socfb-Bowdoin47.mtx & $2 \mathrm{k}$ & 4.94 & 22 & 9 \\
socfb-Brandeis99.mtx & $4 \mathrm{k}$ & 16.74 & 23 & 9 \\
socfb-Brown11.mtx & $9 \mathrm{k}$ & 140.75 & 32 & 10 \\
socfb-Bucknell39.mtx & $4 \mathrm{k}$ & 18.38 & 29 & 9 \\
socfb-CMU.mtx & $7 \mathrm{k}$ & 65.80 & 44 & 15 \\
socfb-Cal65.mtx & $11 \mathrm{k}$ & 180.57 & 50 & 19 \\
socfb-Caltech36.mtx & 769 & 0.38 & 20 & 12 \\
socfb-Colgate88.mtx & $3 \mathrm{k}$ & 15.82 & 33 & 12 \\
socfb-Columbia2.mtx & $12 \mathrm{k}$ & 242.18 & 31 & 10 \\
socfb-Cornell5.mtx & $19 \mathrm{k}$ & 653.97 & 40 & 13 \\
socfb-Dartmouth6.mtx & $8 \mathrm{k}$ & 102.01 & 28 & 11 \\
socfb-Duke14.mtx & $10 \mathrm{k}$ & 229.36 & 33 & 9 \\
socfb-Emory27.mtx & $7 \mathrm{k}$ & 103.40 & 52 & 10 \\
socfb-FSU53.mtx & $28 \mathrm{k}$ & 1346.47 & 54 & 24 \\
socfb-Indiana.mtx & $30 \mathrm{k}$ & 1492.60 & 47 & 11 \\
socfb-Indiana69.mtx & $30 \mathrm{k}$ & 1501.44 & 47 & 11 \\
socfb-JMU79.mtx & $14 \mathrm{k}$ & 240.37 & 38 & 9 \\
socfb-JohnsHopkins55.mtx & $5 \mathrm{k}$ & 31.62 & 44 & 9 \\
socfb-Lehigh96.mtx & $5 \mathrm{k}$ & 34.60 & 37 & 10 \\
socfb-MIT.mtx & $6 \mathrm{k}$ & 62.37 & 32 & 9 \\
socfb-MSU24.mtx & $32 \mathrm{k}$ & 1565.69 & 46 & 11 \\
socfb-MU78.mtx & $15 \mathrm{k}$ & 389.12 & 48 & 11 \\
socfb-Maine59.mtx & $9 \mathrm{k}$ & 90.58 & 28 & 11 \\
socfb-Maryland58.mtx & $21 \mathrm{k}$ & 652.87 & 53 & 9 \\
socfb-Mich67.mtx & $67 \mathrm{k}$ & 8.76 & 27 & 14 \\
socfb-Michigan23.mtx & $30 \mathrm{k}$ & 1539.24 & 44 & 11 \\
socfb-Middlebury45.mtx & $3 \mathrm{k}$ & 11.00 & 24 & 13 \\
socfb-Mississippi66.mtx & $11 \mathrm{k}$ & 262.77 & 47 & 10 \\
socfb-NYU9.mtx & $22 \mathrm{k}$ & 706.92 & 37 & 13 \\
socfb-Northeastern19.mtx & $14 \mathrm{k}$ & 252.21 & 34 & 11 \\
socfb-Northwestern25.mtx & $11 \mathrm{k}$ & 219.91 & 40 & 21 \\
socfb-NotreDame57.mtx & $12 \mathrm{k}$ & 291.54 & 25 & 12 \\
socfb-Temple83.mtx & $14 \mathrm{k}$ & 332.03 & 35 & 10 \\
socfb-Tennessee95.mtx & $17 \mathrm{k}$ & 832.38 & 57 & 9 \\
socfb-Texas80.mtx & $32 \mathrm{k}$ & 2835.07 & 57 & 12 \\
socfb-Texas84.mtx & $36 \mathrm{k}$ & 4410.97 & 50 & 9 \\
\hline & & & & \\
\hline
\end{tabular}

\begin{tabular}{|c|c|c|c|c|}
\hline Dataset & $|V|$ & $t_{1.0}$ & $\omega_{1.0}$ & $\omega_{L B}$ \\
\hline socfb-Trinity 100.mtx & $3 \mathrm{k}$ & 15.25 & 36 & 11 \\
\hline socfb-Tufts18.mtx & $7 \mathrm{k}$ & 122.37 & 34 & 9 \\
\hline socfb-Tulane29.mtx & $8 \mathrm{k}$ & 172.76 & 38 & 16 \\
\hline socfb-UC33.mtx & $17 \mathrm{k}$ & 734.74 & 42 & 15 \\
\hline socfb-UC61.mtx & $14 \mathrm{k}$ & 538.35 & 46 & 18 \\
\hline socfb-UC64.mtx & $7 \mathrm{k}$ & 80.81 & 32 & 13 \\
\hline socfb-UCF52.mtx & $15 \mathrm{k}$ & 593.20 & 59 & 14 \\
\hline socfb-UCLA.mtx & $20 \mathrm{k}$ & 1282.03 & 51 & 9 \\
\hline socfb-UCLA26.mtx & $20 \mathrm{k}$ & 1284.71 & 51 & 9 \\
\hline socfb-UCSB37.mtx & $15 \mathrm{k}$ & 653.25 & 53 & 12 \\
\hline socfb-UCSC68.mtx & $9 \mathrm{k}$ & 160.68 & 30 & 12 \\
\hline socfb-UCSD34.mtx & $15 \mathrm{k}$ & 592.51 & 43 & 12 \\
\hline socfb-UChicago30.mtx & $7 \mathrm{k}$ & 102.23 & 33 & 9 \\
\hline socfb-GWU54.mtx & $12 \mathrm{k}$ & 190.39 & 42 & 9 \\
\hline socfb-Hamilton46.mtx & $2 \mathrm{k}$ & 5.56 & 25 & 15 \\
\hline socfb-Harvard1.mtx & $15 \mathrm{k}$ & 462.97 & 39 & 9 \\
\hline socfb-Howard90.mtx & $4 \mathrm{k}$ & 25.98 & 47 & 9 \\
\hline socfb-UConn.mtx & $17 \mathrm{k}$ & 404.61 & 49 & 9 \\
\hline socfb-UConn91.mtx & $17 \mathrm{k}$ & 405.48 & 49 & 9 \\
\hline socfb-UF.mtx & $35 \mathrm{k}$ & 2156.92 & 55 & 13 \\
\hline socfb-UF21.mtx & $35 \mathrm{k}$ & 2193.94 & 55 & 13 \\
\hline socfb-UGA50.mtx & $24 \mathrm{k}$ & 1153.77 & 51 & 12 \\
\hline socfb-UIllinois.mtx & $31 \mathrm{k}$ & 1663.48 & 56 & 9 \\
\hline socfb-UIllinois $20 . \mathrm{mtx}$ & $31 \mathrm{k}$ & 1656.72 & 56 & 10 \\
\hline socfb-UMass92.mtx & $17 \mathrm{k}$ & 376.00 & 35 & 10 \\
\hline socfb-UNC28.mtx & $18 \mathrm{k}$ & 597.23 & 46 & 11 \\
\hline socfb-UPenn7.mtx & $15 \mathrm{k}$ & 447.09 & 41 & 10 \\
\hline socfb-USC35.mtx & $17 \mathrm{k}$ & 614.39 & 60 & 9 \\
\hline socfb-USF51.mtx & $13 \mathrm{k}$ & 214.36 & 43 & 10 \\
\hline socfb-USFCA72.mtx & $3 \mathrm{k}$ & 4.23 & 28 & 10 \\
\hline socfb-UVA16.mtx & $17 \mathrm{k}$ & 470.91 & 42 & 9 \\
\hline socfb-Vanderbilt $48 . \mathrm{mtx}$ & $8 \mathrm{k}$ & 109.41 & 45 & 9 \\
\hline socfb-Vassar85.mtx & $3 \mathrm{k}$ & 9.47 & 22 & 10 \\
\hline socfb-Vermont70.mtx & $7 \mathrm{k}$ & 50.51 & 38 & 14 \\
\hline socfb-Villanova62.mtx & $8 \mathrm{k}$ & 87.20 & 36 & 9 \\
\hline socfb-Virginia63.mtx & $21 \mathrm{k}$ & 629.39 & 51 & 9 \\
\hline socfb-Wake73.mtx & $5 \mathrm{k}$ & 53.47 & 45 & 10 \\
\hline socfb-WashU32.mtx & $8 \mathrm{k}$ & 114.94 & 41 & 9 \\
\hline socfb-Wellesley22.mtx & $3 \mathrm{k}$ & 7.50 & 27 & 10 \\
\hline socfb-Wesleyan43.mtx & $4 \mathrm{k}$ & 14.84 & 28 & 19 \\
\hline socfb-William77.mtx & $6 \mathrm{k}$ & 66.31 & 38 & 10 \\
\hline socfb-Williams40.mtx & $3 \mathrm{k}$ & 9.44 & 24 & 14 \\
\hline socfb-Wisconsin87.mtx & $24 \mathrm{k}$ & 855.08 & 35 & 12 \\
\hline socfb-Yale4.mtx & $9 \mathrm{k}$ & 153.50 & 30 & 10 \\
\hline
\end{tabular}

Table 2: Results of the TrustCLQ algorithm on Facebook networks. $t_{1.0}$ denotes the running time of the algorithm for $S C A L E=1.0 ; \omega_{L B}$ is the known lower bound on the size of the maximum clique; $\omega_{1.0}$ denotes the solution obtained by the TrustCLQ algorithm for $S C A L E=1.0$

Table 2 shows that the TrustCLQ algorithm allocates maximum cliques of a much larger sizes than the known lower bounds on the sizes of the maximum cliques. We also note that the running time of the algorithm is acceptable for solving problems of the approximate search for the maximum cliques on real social graphs.

\section{Conclusion}

In this paper, an heuristic algorithm for solving the maximum clique search problem is proposed. The algorithm uses both the well-known branch-and-bound method, as well as new ideas that were not previously mentioned by researchers. The algorithm uses an it- 
erative approach for solving $\mathrm{MCP}$ and the results is practically independent of the order of vertices. In addition, it does not use the graph partition into independent sets.

The algorithm was tested on both artificial graphs and real networks. He showed not so high accuracy on DIMACS graphs, which are not real graphs, but are good for testing MCP algorithms. The algorithm showed good results when running on social networks, for which the obtained solution (i.e. the size of maximum clique) exceeded the known lower bound on the size of the maximum clique.

The algorithm was compared with two wellknown algorithms. Each of them is good in individual cases. We can not say that the proposed algorithm is completely better in terms of the MCP solution. However, it works well on some graphs.

The future research may include the comparison of the proposed approach with the state-of-the-art methods described in [12] and [18].

Acknowledgements: The work has been supported by the Ministry of Science and Higher Education of Russian Federation (Project FSRR-2020-0006).

\section{References:}

[1] M.G.C. Resende and C.C. Ribeiro, Optimization by GRASP: Greedy Randomized Adaptive Search Procedures. Springer, 2016.

[2] Q. Wu and Jin-Kao Hao, A review on algorithms for maximum clique problems, European Journal of Operational Research, 242(3), 2015, pp. 693-709.

[3] E. Tomita and T. Seki, An efficient branch-andbound algorithm for finding a maximum clique, In Proceedings of the 4th International Conference on Discrete Mathematics and Theoretical Computer Science, DMTCS'03 2003, pp. 278289, Berlin, Heidelberg, 2003. Springer-Verlag.

[4] E. Tomita and T. Kameda, An efficient branchand-bound algorithm for finding a maximum clique with computational experiments, Journal of Global Optimization 37(1), 2007, pp. 95-111.

[5] E. Tomita, Y. Sutani, T. Higashi and M. Wakatsuki, A simple and faster branch-and-bound algorithm for finding a maximum clique with computational experiments, IEICE Transactions on Information and Systems E96.D(6), 2013, pp. 1286-1298.
[6] J. Konc and D. Janezic, An improved branch and bound algorithm for the maximum clique problem, MATCH - Communications in Mathematical and in Computer Chemistry 58, 2007, pp. 569-590.

[7] M. Depolli, J. Konc, K. Rozman, R. Trobec and D. Janezic. Exact parallel maximum clique algorithm for general and protein graphs, Journal of chemical information and modeling 53(9), 2013, pp. 2217-28.

[8] P. San Segundo, D. Rodriguez-Losada and A. Jimenez, An exact bit-parallel algorithm for the maximum clique problem, Computers \& Operations Research 38, 2011, pp. 571-581.

[9] B. Pattabiraman, Md. Mostofa Ali Patwary, A. H. Gebremedhin, W.-K. Liao and A. Choudhary, Fast algorithms for the maximum clique problem on massive sparse graphs, Eds. A. Bonato, M. Mitzenmacher and P. Prałat, Algorithms and Models for the Web Graph, 2013, pp. 156169, Cham, 2013. Springer International Publishing.

[10] R. A. Rossi, D. F. Gleich, A. H. Gebremedhin and Md. M. A. Patwary, A fast parallel maximum clique algorithm for large sparse graphs and temporal strong components, CoRR, abs/1302.6256, 2013.

[11] P. Daniluk, G. Firlik and B. Lesyng, Implementation of a maximum clique search procedure on cuda, Journal of Heuristics 25(2), 2019, pp. 247-271.

[12] C.-M. Li, H. Jiang and F. Manyà, On minimization of the number of branches in branchand-bound algorithms for the maximum clique problem, Computers \& Operations Research 84, 2017, pp. 1-15.

[13] R. Battiti and M. Protasi, Reactive local search for the maximum clique problem1, Algorithmica 29(4), 2001, pp. 610-637.

[14] W. Pullan and H. H. Hoos, Dynamic local search for the maximum clique problem, J. Artif. Int. Res. 25(1), 2006, pp. 159-185.

[15] W. Pullan, F. Mascia and M. Brunato, Cooperating local search for the maximum clique problem, Journal of Heuristics 17(2), 2011, pp. 181199.

[16] S. Busygin, A new trust region technique for the maximum weight clique problem, Discrete Applied Mathematics 154(15), 2006, pp. 20802096. 
[17] Q. Zhang, J. Sun and E. Tsang, An evolutionary algorithm with guided mutation for the maximum clique problem, IEEE Transactions on Evolutionary Computation 9(2), 2005, pp. 192200.

[18] Y. Wang, S. Cai and M. Yin, Two Efficient Local Search Algorithms for Maximum Weight Clique Problem, Proceedings of the Thirtieth AAAI Conference on Artificial Intelligence (AAAI-16) 2016, pp. 805-811.

[19] Y. Wang, S. Cai, J. Chen and M. Yin, SCCWalk: An efficient local search algorithm and its improvements for maximum weight clique problem, Artificial Intelligence 280, 2020, pp. 103230.

[20] P. S. Segundo, S. Coniglio, F. Furini and I. Ljubic, A new branch-and-bound algorithm for the maximum edge-weighted clique problem, European Journal of Operational Research 278(1), 2019, pp. 76-90.

[21] T. Yu and M. Liu, A memory efficient maximal clique enumeration method for sparse graphs with a parallel implementation, Parallel Computing 87(1), 2019, pp. 46-59.

[22] L. Chang, Efficient maximum clique computation and enumeration over large sparse graphs, The VLDB Journal 2020, https://doi.org/10.1007/s00778-020-00602-z

[23] P. S. Segundo, F. Furini and J. Artieda, A new branch-and-bound algorithm for the Maximum Weighted Clique Problem, Computers \& Operations Research 110, 2019, pp. 18-33.

[24] Y. Chu, B. Liu, S. Cai, C. Luo and H. You, An efficient local search algorithm for solving maximum edge weight clique problem in large graphs, Journal of Combinatorial Optimization 2020, https://doi.org/10.1007/s10878020-00529-9

[25] B. Nogueira and R.G.S. Pinheiro, A GPU based local search algorithm for the unweighted and weighted maximum s-plex problems, Ann. Oper. Res. 284, 2020, pp. 367-400.

[26] E. Sevinc and T. Dokeroglu, A novel parallel local search algorithm for the maximum vertex weight clique problem in large graphs, Soft Comput. 24, 2020, pp. 3551-3567.

[27] H. Jiang, C.-M. Li, Y. Liu and F. Manya, A Two-Stage MaxSAT Reasoning Approach for the Maximum Weight Clique Problem, Proc. of Thirty-Second AAAI Conference on Artificial Intelligence 2018, pp. 1338-1346.
[28] Y. Wang, S. Cai and M. Yin, New heuristic approaches for maximum balanced biclique problem, Information Sciences 432, 2018, pp. 362375.

[29] D. V. Andrade, M. G. C. Resende and R. F. Werneck, Fast local search for the maximum independent set problem, Journal of Heuristics 18(4), 2012, pp. 525-547.

[30] L. A. Sanchis, Generating hard and diverse test sets for np-hard graph problems, Discrete Applied Mathematics 58(1), 1995, pp. 35 - 66.

[31] M. Batsyn, B. Goldengorin, Eu. Maslov and P. Pardalos, Improvements to mcs algorithm for the maximum clique problem, Journal of Combinatorial Optimization 27, 2014, pp. 397-416.

[32] R. A. Rossi and N. K. Ahmed, The network data repository with interactive graph analytics and visualization, Proceedings of the TwentyNinth AAAI Conference on Artificial Intelligence, 2015, pp. 4292-4293. 